\title{
An Unusual Cause of Insomnia
}

Tauseef Afaq, M.B.B.S.; Ulysses J. Magalang, M.D.; Aneesa M. Das, M.D.

23-year-old African American male was referred - for sleep maintenance insomnia for the past several years. He complained of occasional morning headaches. He denied snoring, hypnagogic hallucinations, sleep paralysis, and cataplexy. His Epworth Sleepiness Scale score (ESS) was 12/24.

At age 16, he was diagnosed with a posterior fossa medulloblastoma (T3) and underwent surgical resection, radiation, and chemotherapy and has had no evidence of recurrence on recent brain magnetic resonance imaging. He has no history of taking any medications known to affect the central nervous system, including narcotics/opioids. His body mass index was $18 \mathrm{~kg} / \mathrm{m}^{2}$, and he had mild slurred speech. His oropharynx was normal. His oxygen saturation while breathing room air was $91 \%$.

His room air arterial blood gas (ABG) that was performed because of the relatively low oxygen saturation showed a $\mathrm{pH}$ of $7.38, \mathrm{pCO}_{2}$ of $57 \mathrm{~mm} \mathrm{Hg}$, and $\mathrm{pO}_{2}$ of $65 \mathrm{~mm} \mathrm{Hg}$. His chest $\mathrm{x}$-ray was normal. He had no polycythemia, and echocardiography findings were normal.

QUESTION: What is the cause of this patient's insomnia? 
ANSWER: Central sleep apnea (CSA), likely due to impaired central drive. This patient has hypercapnic CSA and a presumed blunted ventilatory drive as a result of his previous brain surgery and radiation for medulloblastoma.

\section{DISCUSSION}

PSG (scored using the American Academy of Sleep Medicine recommended criteria) revealed poor sleep efficiency of $45 \%$ with a total of 80 central apneas and 17 hypopneas, and an overall apnea-hypopnea index (AHI) of 33/h (NREM AHI: 16/h, REM AHI 99/h). The overall arousal index was 31.2/h. A fragment of his PSG is shown in Figure 1.

The patient's ABG showed chronic hypercapnia without increased alveolar-arterial (A-a) oxygen gradient, which would be consistent with hypoventilation due to drive or weakness rather than lung disease. Pulmonary function testing revealed normal spirometry and lung volumes. Normal spirometry would be expected in hypercapnia due to ventilatory drive. Because he had difficulty following the instructions for the measurements of maximum inspiratory (MIP) and expiratory pressures (MEP) which registered low values, diaphragmatic electromyography was performed, which showed normal bilateral phrenic nerve responses and normal needle electrode examination of the right hemi-diaphragm. There was no evidence of excessive dead space ventilation as a contributing factor to chronic hypercapnia with a $\mathrm{Vd} / \mathrm{Vt}$ of 0.36 that was determined at rest. Although a formal hypercapnic ventilatory response was not performed, his work up has virtually eliminated other potential causes of chronic hypercapnia and was presumed likely due to a blunted ventilatory drive. He was treated with nocturnal noninvasive ventilation using a bilevel machine in the spontaneous/timed mode with improvements in sleep efficiency and AHI.

It is important to remember that insomnia has a variety of causes, and CSA has to be considered should the history suggest the possibility. Previous studies have reported that brainstem tumors are associated with CSA, before and even after surgical resection. ${ }^{1,2}$ Our case is unique in that a predominant presenting complaint of insomnia has not been reported in patients who have undergone surgical resection of brainstem tumors with consequent CSA.

Figure 1-PSG showing numerous episodes of central sleep apnea (arrows) during Stage R (REM) sleep

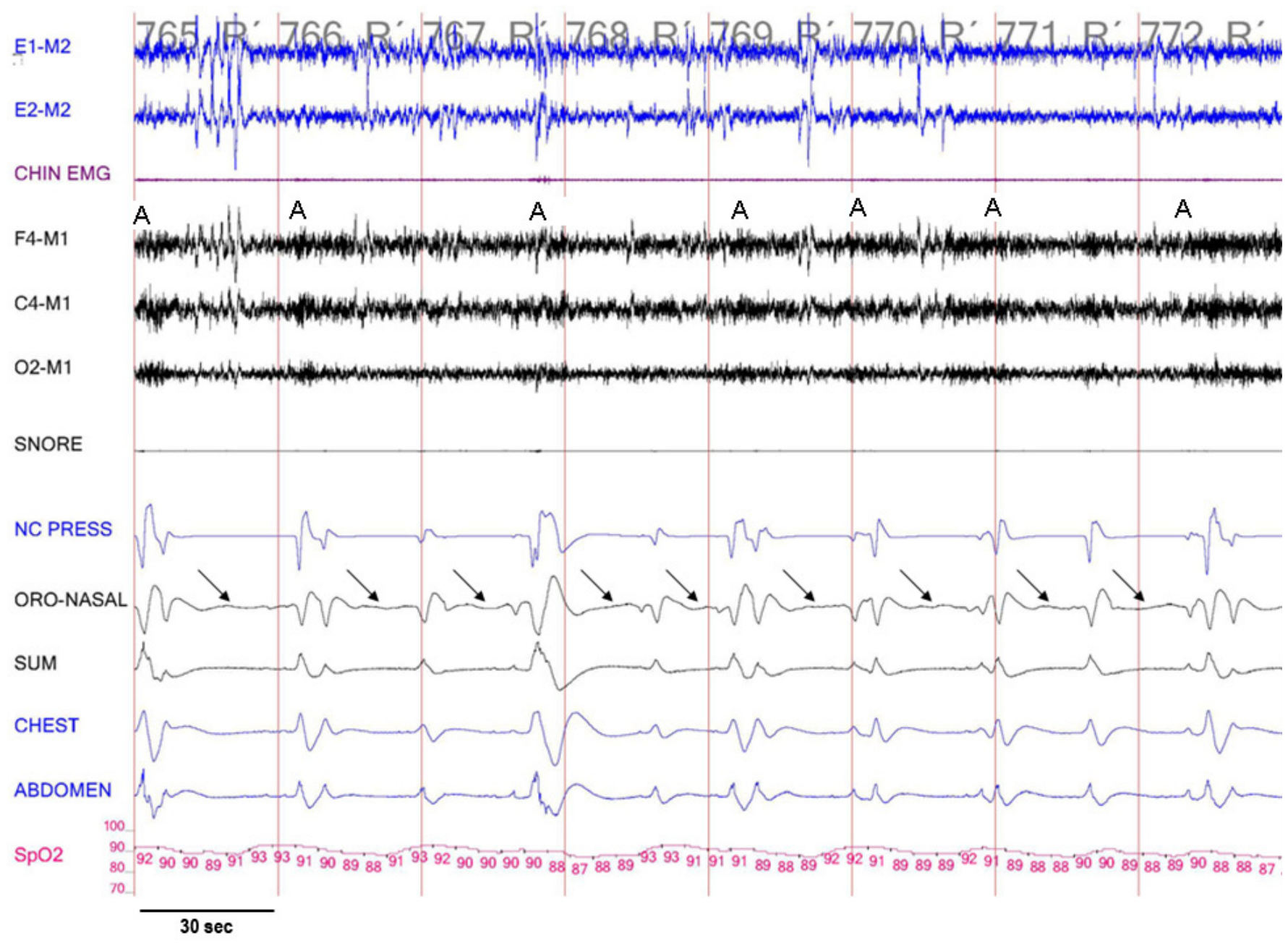

A, arousal; Snore, snore microphone; NC Press, nasal cannula pressure transducer; Oro-Nasal, oro-nasal flow using thermocouple; Chest/Abdomen, chest and abdomen inductance plethysmography; $\mathrm{SpO}_{2}$ oxygen saturation using pulse oximetry. 


\section{PEARLS}

1. CSA should be suspected as a cause of insomnia in a patient who has had resection of posterior fossa tumors.

2. In those with a blunted ventilatory drive, $A B G$ values show chronic hypercapnia with a normal $\mathrm{pO}_{2}(\mathrm{~A}-\mathrm{a})$ gradient. Other causes of hypercapnia should be looked for.

3. Brainstem tumors, even after successful resection, have been associated with CSA.

4. Treatment of hypercapnic CSA includes nocturnal noninvasive ventilation.

\section{CITATION}

Afaq T; Magalang UJ; Das AM. An unusual cause of insomnia. J Clin Sleep Med 2012;8(5):623-625.

\section{REFERENCES}

1. Ioos C, Estournet-Mathiaud B, Pinard JM, Cheliout-Héraut F. Sleep disorders caused by brainstem tumor: case report. J Child Neurol 2001;16:767-70.

2. Manning HL, Leiter JC. Respiratory control and respiratory sensation in a patient with a ganglioglioma within the dorsocaudal brain stem. Am J Respir Crit Care Med 2000:161:2100-6.

\section{SUBMISSION \& CORRESPONDENCE INFORMATION}

Submitted for publication September, 2011

Submitted in final revised form January, 2012

Accepted for publication February, 2012

Address correspondence to: Aneesa M. Das, M.D., 201 Davis Heart and Lung

Research Institiute, Division of Pulmonary, Allergy, Critical Care, and Sleep Medicine,

The Ohio State University Medical Center, 473 West 12th Avenue, Columbus OH

43210; Tel: (614) 293-4062; Fax: (614) 293-6340; E-mail: aneesa.das@osumc.edu

\section{DISCLOSURE STATEMENT}

This was not an industry supported study. The authors have indicated no financial conflicts of interest. 